III.

A.

Ms. de la Biblioteca del Escorial.

\section{REUELAÇION DE VN HERMITANNO.}

[Fol. I 29. v.] Esta es vna reuelaçion que acaesçió a vn omn e bueno, hermitanno de santa vida, que estaua resando vna noche en su hermita é oyó esta reuelaçion, el qual luego la escriuió en rymas, ca era sabidor en esta çiençia gaya.

\section{Comiença é dise asy:}

I.

Despues de la prima la ora pasada, en el mes de enero la noche primera, en cccc. e veynte durante la hera, estando acostado allá en mi posada; non pude dormir essa trasnochada, á la mannana un suenno me vino, veredes, sennores, lo que me avino mientra pasaua el alumbrada.

II.

[Fol. 130.] En vn valle fondo, escuro, apartado,

espeso de xaras, sonné que andaua 10 buscando salida é non la fallaua, tope con un omne que yasia fynado. Holia muy mal, ca estaua fynchado, los ojos quebrados, la fas denegrida, la boca abierta, la barba cayda, de gusanos é moscas muy acompannado.

\section{III.}

Mirando el cuerpo de chico valor, oy vna bos aguda muy fierra, abri los mis ojos por mirar quien era, vy vna aue de blanca color. Desía contra el cuerpo: hereje, traydor,
B.

Ms. de la Biblioteca Nacional de Paris

- No. 313 (ant. 8168) - S. XV.

[Fol. 179. v.] DISPUTA DEL CUERPO E DEL ANIMA. 1
I.

Despues de la primera hora passada en el mes de enero la noche primera ${ }^{2}$ quatro cientos e veynt entrante la era ${ }^{3}$ estando acostado en una posada non pude dormir essa trasnochada vino me un suenno alla al maytino ${ }^{5}$ direuos señores lo que me avino ${ }^{6}$ fasta que paso toda el aluorada. II.

En un valle fondo, escuro apartado ${ }^{7}$

espesso de xaras sonné que andaua buscando salida e non la fallaua ${ }^{8}$ topé con un cuerpo que estaua finado 9 Olia muy mal seya finchado 10 los ojos quebrados la faz denegrida " I5 la boca abierta la barba cayda de muchos gusanos bien acom-

\section{III.} pan̄ado 12

Mirando aquel cuerpo de chica valor ${ }^{13}$ oy una voz aguda muy fiera alçé los mis ojos por ver de quien era 14 20 ví venir un aue de blanca color ${ }^{15}$ deziendo contra el cuerpo ereje traydor ${ }^{10}$

C. - Ms. de la Bibl. Nac. de Paris - No. 230 (ant. 7825) - S. XVI. ${ }^{1}$ [Fol. 225]. Disputa del anima e del cuerpo. 2 e. e. m. d. janero .... 3 .... ve viente... n. puede d. esta transnochanda. 5 v. m. en s. a. el matino. ${ }^{6}$ diremos senyores 1 . q. á mi a. ${ }^{7} \mathrm{E}$. hun v. escuro fundo a. $8 \mathrm{~b}$. sallida e... ${ }^{9}$ t. c. hun c ..... ${ }^{10}$ Olya m. m. s. fincado. ${ }^{11} \mathrm{~L}$. oios q. 1. fas d. ${ }^{12} \mathrm{~d}$. m. guzanos b. acompanyada. ${ }^{13} \mathrm{M}$. aquell c. d. xicha $v$. " alce l. mys oios p.... 15 v. v. hun a. d. blancha c. ${ }^{16}$ dize c. e. c. ereie t. 
del mal que fesiste, si eres repiso, por tu vana-gloria e falso riso, yo enel infierno biuo con dolor.

IV.

Asentóse muy paso asu cabeçera

çercando el cuerpo todo aderredor. batiendo las alas con muy grand dolor. fasía grand llanto de estranna manera : desia cuytada, commo soi sennera non fallo lugar do pueda guarir, malo fué el dia que oue avenir aser tu çercana e tu conpannera.

V.

De Dios nin del mundo pauor non oviste,

falsaste su ley e sus mandamientos, yncrédulo fueste en tus pensamientos, jurando en bano mentiste, falsaste. Apobres cuytados lo suyo tomaste 45 con tu luxuria e mucha cobdiçia, e con tu soberuia e grande abariçia, donde yo era linpia muy mal me ensusiaste. del mal que feziste si eras rep'so ${ }^{1}$ por tu vana gloria e muy poco seso ${ }^{2}$ jamas nel infierno viuiré $\bar{e}$ dolor $^{3}$ IV.

25 [Fol. I 80.] Asentóse queda á su cabeçera ${ }^{4}$

e anduuo el cuerpo todo èderredor 5 batiendo las alas con muy grā temor ${ }^{6}$ faziendo llanto de estranna manera? deziendo cuytada como soy sennera

30 non fallo lugar do pueda guarir escuro fué el dia que oue a venir á ser tu çercana e tu compannera. $\mathrm{v}$.

En tanto que puedo agora aquí estar quiero fablar un poco contigo 9

35 bien sabes tu el mi mal amigo 10 que por tus errores e tu mal vsar pecaste e feziste a muchos pecar ${ }^{11}$ de lo qual repriso ser non $\bar{q}$ siste ${ }^{12}$ e avn penitēçia jamas non ouiste. ${ }^{13}$

40 por que yo mezquina avre de lazdrar. ${ }^{14}$ VI.

De dios e del mūdo pauor perdiste ${ }^{15}$ pasaste su ley e sus mandamientos ${ }^{16}$ incrédulo fuste en tus pensamientos ${ }^{17}$ juraste en vano falsaste e mentiste is A pobres cuytados sienpre corriste ${ }^{19}$ por tu grā gula luxuria auariçia 20 açidia homiçidia inuidia cobdiçia 21 do yo era linpia grā mal me feziste 22

VII.

[Fol. 180. v.] Ado tus moradas do es tu arreo 23

50 tu oro e tu plata e tu grā aver 24

1...... s. e. represo. 2 [Fol. 225. v.] . . e. m. p. ceso. 3 j. en i. v. con d. Assentose muy q... ${ }^{5} \mathrm{e}$ andouo el c. $t$. enderrador. 6 .... m. grand t. ${ }^{7}$ f. lanto d... ${ }^{8}$ a. s. t. cercana ... ${ }^{9}$ q. f. hun ... 10....t. e. my .... ${ }^{11}$ peccaste e f. a m. peccar. ${ }^{12} \mathrm{~d}$. 1. q. represo esser n. queziste. ${ }^{13} \mathrm{e}$ a. penitencia j. n. ovieste. ${ }^{11} \mathrm{p} . \mathrm{q} . \mathrm{y}$. mesquina haure d. lazerar. ${ }^{15} \mathrm{Del} \mathrm{d}$. e d. mondo. ${ }^{16}$ passaste ... ${ }^{17} \mathrm{i}$. fueste ... ${ }^{18} \mathrm{j}$. in v. falçaste . . ${ }^{19}$ [Fol. 226] . . c. siempre c. ${ }^{20}$ p. t. grand g. l. e auaricia. 21 Falta este verso en C. ${ }^{22}$ d. y. e. limpia grand $\mathrm{m} . .{ }^{23} \mathrm{~A}$. tu $\mathrm{m}$. d. e. tus arreos. $24 \ldots .$. e t. grand hauer.

22 Repiso, arrepentido. aislada. 40 Lazdrar, lacerar.
23 Riso, risa, burla. 29 Sennera, sola 47 Açidia, flojedad pereza. 
tus joyas muy ricas e tu grā poder ${ }^{1}$ do es tu graçia ado tu asseo ${ }^{2}$ Do es tu argullo ado tu meneo ${ }^{3}$ mira agora que fué todo nada 55 todo fizo fin en una braçada 4 de tierra en que estas segū que ora veo."

VI.

[Fol. 131.] Responde-me agrora á esto que te digo, que tu bien solias de ty dar rrason, pues mira agora mi tribulaçion, que en alto nin en baxo non fallo abrigo. 60 Commo enmudesçiste mortal enemigo delo que solias fablar é desir, mas me valdria contigo morir que non perseguir aquesto que sygo. Dise el cuerpo:

VII.

Essa ora el cuerpo fiso mouimiento, 65 alçó la cabeça, començó á fablar, e dixo : sennora ¿ por que tanto culpar me quiere agora syn meresçimicnto? Que sy dixe ó fise fué por tu talento, sy non mira agora qual es my poder, 70 que estos gusanos non puedo toller,

que comen las carnes de mi criamiento.

VIII.

Tu mi sennora, yo tu scruidor,

mis piés y manos por ty se mouieron,

á do quesiste alli anduuicron, yo fui la morada, tu el morador. Pues por que me cargas la culpa $\mathrm{c}$ error
VIII.

Responde me agora a esto que te digo ${ }^{6}$

ca tu bicn solias de ti dar razon ${ }^{7}$ non cates agora mi tribulaçion ${ }^{8}$ quen alto nin $\bar{e}$ baxo non fallo abrigo ${ }^{9}$ Como mudeçiste mortal enemigo 10 de lo que solias fablar e dezir mas me valdria contigo morir " que non seguir aquesto que sigo.

Respuesta del cuerpo. ${ }^{12}$

IX.

El cuerpo essa hora fizo mouemiento ${ }^{13}$ abrió su boca pensó de fablar 14 e dixo scñora por que me culpar ${ }^{15}$ quieres agora sin meresçemiento ${ }^{16}$ Ca si dixe ó fize fué por tu talento $\sin \overline{0}$ ves agora quanto es mi poder ${ }^{17}$ que aquestos gusanos non puedo toller ${ }^{18}$

que comen las carnes del mi criamento ${ }^{19}$

$\mathrm{X}$.

[Fol. I8I.] Tu mi señora e yo tu seruidor 20

mis pies e mis manos por ti se mouicron 21

75 ado tu mandaste allá anduuieron 22 yo era la morada es tu el morador. Pues por que me cargas la culpa e error ${ }^{23}$

1.... e t. grand p. $2 \ldots$ t. gracia do es t. a. $3 \ldots$ t. vergullo ado es t. m. 4 t. f. fyn e. u. brassada. ${ }^{5}$..... q. c. segund q. o. v. ${ }^{6}$ Respuende $\mathrm{m}$. a. a cste que digo. ${ }^{7}$.... d. ty d. ${ }^{8} \mathrm{n}$. caytas a. my tribulacion. " q. a. ny b. n... ${ }^{10}$ Como enmudeciste $\mathrm{m}$. e. ${ }^{11} \mathrm{~m}$. $\mathrm{m}$. valiera c. $\mathrm{m}$. 12 R. que faze el c. $13 \ldots$... f. mouimiento. ${ }^{14}$ [Fol. 226. v.] A. la s. b. començo d. f. ${ }^{15} \mathrm{e}$ d. senyora p. q... ${ }^{16} \mathrm{q}$. a. syn merescimiento. ${ }^{17}$ Syno v. a. q. e. my p. ${ }^{18} \mathrm{q}$. essos guzanos n. p. t. ${ }^{10} \ldots$. d. mi c. ${ }^{20} \mathrm{q}_{\mathrm{my}}$ senyora e yo t. s. 21 Mys p. e mys m. p. ty s. m. $22 . . . \mathrm{m}$. a. andouieron. 23 ... 1. c. y error.

7 I Toller, quitar.

7eitschr. f. rom. Ph. JI. 
en caso que algo yo cobdiçié aber, la fuerça, sennora, en ty fué e poder, por que me dexaste conplir mi sabor. 80

Dise el anima:

IX.

O cuerpo maldito, vil, enconado, lleno de fedor e de grand calabrina 90 metieron te en foyo, cubrieron te ayna, dexaron te dentro amal de tu grado. Por ende tu piensas que as ya librado,

primero serás delante el derecho, donde darás cuenta de todo tu fecho 95 que enel mundo fesiste, do poco

has durado.

\section{$\mathrm{X}$.}

[Fol.1 32.] Dyme agora, cuerpo de grand trayçion, por que desuarias en tu departir, que sy tu quisieses la verdat desir, bien sabes por çierto qual fué la 100 ocasyon.

Tres contrarios malos de vna condiçion,

el mala del mundo tan falaguero, puesto que algo yo cobdiçié 1 pues poder entero señora en ti fue ${ }^{2}$ por que me dexauas cōplir mi sabor ${ }^{3}$

$\mathrm{XI}$.

Agora non puedo contigo alongar ca non he lugar nī tengo sazon ${ }^{4}$ seg $\bar{u}$ me paresçe non as defension ${ }^{5}$ nin por tu sentençia non quiero pasar ${ }^{6}$

85 Ve te por dios e dexa me estar ${ }^{7}$ non me persiguas que asaz padesco $^{8}$ si dizes que yo la culpa merezco ${ }^{9}$ non dexes si puedes de me la cargar ${ }^{10}$ Replicato del anima."

$\mathrm{XV}$.

Cuerpo maldicto lixoso enconado 12 por el grā fedor de tu fedentina ${ }^{13}$ metieronte en foya cobrieron te ayna dexaron te dentro a mal de tu grado 14 $\mathrm{Tu}$ piensas por tanto que as ya librado 15

comigo as de ser avn a derecho ${ }^{16}$ avras gualardon de todo tu fecho ${ }^{17}$ nel infierno por siēpre seras cōdenado 18

Replicato del anima. ${ }^{19}$

$\mathrm{XII}$.

Cuerpo maldicto de grā trayçion ${ }^{20}$ como desuarias en tu departir ${ }^{21}$ ca si tu quesieses la verdat dezir ${ }^{22}$ bien sabes qual fue la mi entinçion ${ }^{23}$ Tres contrarios de mala perdiçion 24 fezistes en mi muy grā dannamiento ${ }^{25}$
1... a, y cobdicie. 4... h. luga nyn t. s. sentencia n. q. passar. ${ }^{9}$ sy d.... l. c. meresco. 2... e. senyora . 3 ... m. de complir my $\mathrm{s}$. ${ }^{5}$ segund $\mathrm{m}$. parece $n$. has deffencion. ${ }^{6}$ ny p. $t$. 7 ...d. e. dexe m. e. ${ }^{8}$ n. m. persigas q. assaz p. 10 [Fol. 227.] 11 Replica la anima. ${ }_{12} \mathrm{C}$. maldito liro e. ${ }^{13}$ p. e. grand ... ${ }^{14}$ d. t. d. mal d .... ${ }^{15}$... q. has y . ${ }^{16}$ Conmigo .... ${ }^{17}$ averas golardon ... ${ }^{18}$ por siempre en inferno $\mathrm{s}$. condemnado. ${ }^{19}$ Replica lanima. $\quad 20 \mathrm{El} \mathrm{c.} \mathrm{maldito} \mathrm{d.} \mathrm{grand} \mathrm{traycion.} 21$ c. desuanas ... $2.2 \ldots t$. quesiesses ... $23 .$. l. my intencion. $24 \ldots$ d. $\mathrm{m}$. pedicion. 25 feziestes e. my $m$. grand $d$.

89 Hemos alterado la colocacion de la copla XV. de los mss. de Paris, poniéndola al frente de la que les corresponde en el de el Escorial. 90 Calabrina, hedor. 89 Replicato, respuesta, réplica. 89 Lixoso, de lixo, cosa vil, inmunda. 
el diablo maldito, e tu el primero, traxiste-me atada en tu prision.

Dise el cuerpo:

$\mathrm{XI}$.

Por que, sennora, mas enojar me quieres agora en esta sason, que en quanto dexiste non tienes rason

vete en buena ora, dexes-me estar. Pues el sennor nos ha de jusgar e dará á cada vno su meresçimiento mas bien me paresçes que eres çimiento

pues por tus malos fechos has de 120 por tanto tu mesma te deues juzgar 14 penar

\section{XII.}

Ellos estando en esta porfia salió vn diablo negro de vn espesura, gesto espantable, de mala figura, el diablo el mundo e tu el çimiento ${ }^{1}$ trayades me puesta en vuestra p'sion. ${ }^{2}$ XIII.

I05 [Fol. I8I. v.] Non auia lugar de me defender ${ }^{3}$

con tales contrarios que me perseguian

yo yua sin grado do ellos querian pues al non podia con ellos fazer.4 $E$ tu con tu acuçia e tu bollesçer 5

I IO cargaron a mi de carga complida 6 por lo qual agora non fallo guarida $\overline{\mathrm{n}}$ fallo lugai do pueda estorçer ${ }^{7}$

Replicato del cuerpo. ${ }^{8}$

XIV.

Por justa razon te quiero prouar que fuste causa de todo pecado ${ }^{9}$ ca desque la muerte nos ouo apartado

falle sin embargo mi propio lugar. ${ }^{10}$ Tu nunca podiste aver $\overline{\mathrm{n}}$ fallar ${ }^{11}$ lugar $\mathrm{n} \overline{1}$ manera para guaresçer ${ }^{12}$ nī te aprouecha ya rebollescer ${ }^{13}$

1...e. cimiento. 2 traydes my p. e. v. presion. ${ }^{3} \mathrm{~N}$. hauia $1 . \mathrm{d} . \mathrm{m}$. deffender. ${ }^{4} \mathrm{p}$. el $\mathrm{n} . . .{ }^{5}$... c. acucia e t. bollecer. ${ }^{6} \mathrm{c}$. a my .... ${ }^{7}$ ny $\mathrm{f} . .$. .. p. estorcer. ${ }^{8}$ [Fol. 227. v.] Replica el cuerpo. ${ }^{9} \mathrm{q}$. fueste $\mathrm{c}$. d. t. peccado. $10 .$. e. my proprio llugar. $11 \ldots$ p. hauer nyn $f .{ }^{12}$ logar ny $m$. p. guarecer. 13 nyn t. a. tu ensoberuecer. $14 \ldots$. t. d. judgar. El ins. B. termina aqui con la copla XV. - En el C. siguen d la XV. las dos coplas siguientes, de las que, la XVI. corresponde a la XII. del ms. A. XVI.

Estando mirando esta porfia sallio hun diablo de una espessura, mortal espantoso de fuerte figura tenezas de fuego en la mano traya. [F. 228.] E dixo contra el aue vos sodes mia conmigo yredes a ver my posada donde seredes por siempre heredada alla fallades assaz grand companya. Fin.

\section{XVII.}

El aue essa hora dio grand apelido e dixo dios myo tu que me criaste rey de piedat libra me deste senyor pues tenes el poder complido. Tomala el dyablo con ella ha fuydo. con el grand pesar luego desparte del suenno que vi pasmado finque ayna perdiera todo my sentido. librarse.

109 Acuçia, solicitud, diligencia, presteza.

112 Estorcer, escapar, 
tynasas de fierro en las manos traya. Dixo contra el ánima: tu serás mia, 125

e conmigo yras allá á mi posada, adonde serás bien aduergada, que allá fallarás asás conpannia.

\section{XIII.}

El angel de Dios que esto beya, fué contra el malo muy ayrado, 130 e dixo: diablo sey yá pagado de quanto mal fases de dia en dia. Pues te atreues con grande osadia, de mi tu yras mal baratado, aunque te pese á mal de tu grado 135 aquesta ánima será toda mía.

\section{XIV.}

[Fol. 133.] Quando fué el anima de pena librada

e vió que tenia tan grand sennorio, dixo del mundo que era desuario, pues que del yba tan despagada. 140 $\mathrm{E}$ dixo asy: mundo, de aquesta begada

yo diré las cosas todas que ay en ty, por que en mi cuytada bien la sentí por donde a poco fuera condenada.

$$
\text { XV. }
$$

Dixo: mundo falso, de grand 145 mesquindad,

bil, reboltoso, de boca balia, jusgo por loco quien mucho en tí fia, nin fas su thesoro de tu vanidad.

Que en caso que pongas en grand potestad

á algunos, en punto trastorna 150 tu rrueda,

non ha tan discreta lengua que pueda desir tus locuras e grand falsedad.

\section{XVI.}

Aquel que ama la tu voluntad todo es lleno de mucha maliçia, soberuia, enbidia e grand 155 abariçia

syenbras en todos e mūcha maldad. Cobdiçia, e gula, e grand torpedad, luxuria muy fea e bil bana-gloria, toda está llena tu mala memoria, de mucha ynfynta e grand 160 banidad.

XVII.

Segund mi juysio son ygnorantes aquellos que syguen la tu falsa via, e tienen fiança en ti cada dia en tus ximonias poco durantes. Que puesto que sean asás 165 abastantes, de mucha riquesa e grand sennorio, todo es niebla, viento e roçio que pasa e corre por sus temporantcs. XVIII.

[Fol. I 34.] A cueruos, milanos, mochuelos cuytados

en alto treuol beo que los subes 170 con tan firmes alas fasta las nubes jamás nunca çesan sobir sus estados. Nobles girafaltes, bayles y sardos derribas e abaxas en mar muy profundo:

los tales juysios de falso mundo 175 . ¿Quien los jusgará por bien hordenados?

XIX.

Aquellos serán bien abenturados que se guardaran de tus fallimientos, de tus enemigas e acaesçimientos feos, torpes e desuariados. I 80 Non podrian ser memorados tus teptaçiones é desuarios, tus symonias, potipas e brios, todos son nada en cabo tornados. XX.

Veo que rreyes e enperadores, 185 Papas, maestres e cardenales, sus mgnifiçencias e pontificales. todos feneçen en vanos sabores. Condes, duques, obispos, priores, segund obraren, ansy gosarán, I 90 e los letrados entonçe verán los malos juysios tornar en sabores. XXI.

Ca sea berdad muy clara paresçe que omne nasçido non ha de leuar

164 Ximonias, simonias. I83 Potipas: desconozco esta palabra. 
de ty falso mundo, sy non bien 195

obrar, que todo lo otro ayna fallesçe.

$¿$ Pues qual peccador non se abborresçe

de syenpre pecar commo fase el moro?

Aquel es que bien obra que fas tu tesoro,

adonde por syenpre el nunca 200

\section{XXII.} peresçe.

[Fol. I 35.] Tú persona que as demandar vasallos y tieira, riquesas $\mathrm{y}$ aver, e non lo rrepartes segund es menester, mas syenpre punas de thesorar;

guardate, mesquino, de mas 205 ofensar,

al tu fasedor con amas las manos, fecho de tierra, monton de gusanos, non quieras por poco perder buen

\section{XXIII.} lugar.

Quanto amorio (nos) quiso mostrar el fijo de Dios por nos redemir, 210 que puso sur cuerpo bendicto á sofrir tan afliçiones esquiuas syn par, e muy de grado quiso tomar muerte cruel e ser flagelado, preso, ferido e muy desonrrado 215 en quanto humano por te saluar.

XXIV.

Non fué meresçiente segund me semeja,

mas fué pastor de grand caridad, que con mucha paçiençia e grand humildat derramó su sangre por ti su oveja. 220 - Muy cara le cuesta la tu pelleja ala su bendicta carne humana, pues pecador con voluntad sana, deues creer á quien bien te conseja. XXV.

Aquella palabra deues noctar 225 que su sancta Iglesia te dise e atisa, reconósçete, hermano, que eres çenisa, e en çenisa te has de tornar: Ca non sabes el dia que te ha dellamar que bayas dar cuenta de quanto 230 fesiste, e sy condepnado ser meresçiste Chino nin Bartolo non cale alegar.

206 Amas, ambas, las dos. 232 Caler, importar, interesar, convenir.

José M. Octavio de Toledo. 